\title{
AN ITERATIVE ALGORITHM BY VISCOSITY APPROXIMATION METHOD FOR MIXED EQUILIBRIUM PROBLEMS, VARIATIONAL INCLUSION AND FIXED POINT OF AN INFINITE FAMILY OF PSEUDO-CONTRACTIVE MAPPINGS
}

\author{
PHAYAP KATCHANG \\ Department of Mathematics, Faculty of Science \\ King Mongkut's University of Technology Thonburi (KMUTT) \\ Bangmod, Bangkok 10140, Thailand \\ E-mail: p.katchang@hotmail.com \\ POOM KUMAM \\ Department of Mathematics, Faculty of Science \\ King Mongkut's University of Technology Thonburi (KMUTT) \\ Bangmod, Bangkok 10140, Thailand \\ Centre of Excellence in Mathematics, CHE, Si Ayutthaya Rd., Bangkok 10400, Thailand \\ E-mail: poom.kum@kmutt.ac.th
}

\begin{abstract}
The purpose of this paper is to investigate the problem of finding a common element of the set of solutions for mixed equilibrium problems, the set of solutions of the variational inclusion problems for inverse strongly monotone mappings and the set of common fixed points for an infinite family of strictly pseudo-contractive mappings in the setting of Hilbert spaces. We prove the strong convergence theorem by using the viscosity approximation method for finding the common element of the above four sets. Our results improve and extend the corresponding results of Peng and Yao [Math. Comput. Modelling 49 (2009), 1816-1828], Plubtieng and Sriprad [Fixed Point Theory Appl. 2009, Article ID 567147] and some well-known results in the literature.
\end{abstract}

2010 Mathematics Subject Classification: Primary 46C05, 47D03, 47H09, 47H10, 47H20.

Key words and phrases: Strong convergence, nonexpansive mapping, fixed point, variational inclusion, mixed equilibrium problem, viscosity approximation method, pseudo-contractive mappings.

The paper is in final form and no version of it will be published elsewhere. 
1. Introduction. Throughout this paper, we assume that $H$ is a real Hilbert space with inner product and norm which are denoted by $\langle.,$.$\rangle and \|$.$\| , respectively, C$ is a closed convex subset of $H, \mathbf{R}$ is the set of real numbers and $\mathbf{N}$ is the set of natural numbers. A mapping $T: C \rightarrow C$ is called nonexpansive if $\|T x-T y\| \leq\|x-y\|$, for all $x, y \in C$. We use $F(T)$ to denote the set of fixed points of $T$, that is, $F(T)=\{x \in C: T x=x\}$. Recall that a self-mapping $f: C \rightarrow C$ is a contraction on $C$ if there exists a constant $\alpha \in(0,1)$ and $x, y \in C$ such that $\|f(x)-f(y)\| \leq \alpha\|x-y\|$. Let $B$ be a strongly positive bounded linear operator on $H$, that is, there is a constant $\bar{\gamma}>0$ with the property

$$
\langle B x, x\rangle \geq \bar{\gamma}\|x\|^{2} \text { for all } x \in H \text {. }
$$

Let $\varphi: C \rightarrow \mathbf{R} \cup\{+\infty\}$ be a proper extended real-valued function and $F$ be a bifunction of $C \times C$ into $\mathbf{R}$. Ceng and Yao [CY] considered the mixed equilibrium problem for finding $x \in C$ such that

$$
F(x, y)+\varphi(y) \geq \varphi(x) \text { for all } y \in C .
$$

The set of solutions of (2) is denoted by $\operatorname{MEP}(F, \varphi)$. We see that $x$ is a solution of problem (2) which implies that $x \in \operatorname{dom} \varphi=\{x \in C \mid \varphi(x)<+\infty\}$. If $\varphi=0$, then the mixed equilibrium problem (2) becomes the following equilibrium problem: find $x \in C$ such that

$$
F(x, y) \geq 0 \text { for all } y \in C .
$$

The set of solutions of $(3)$ is denoted by $E P(F)$. Given a mapping $T: C \rightarrow H$, let $F(x, y)=\langle T x, y-x\rangle$ for all $x, y \in C$. Then $z \in E P(F)$ if and only if $\langle T z, y-z\rangle \geq 0$ for all $y \in C$, i.e., $z$ is a solution of the variational inequality. The mixed equilibrium problems include fixed point problems, variational inequality problems, optimization problems, Nash equilibrium problems and the equilibrium problem as special cases. Numerous problems in physics, optimization and economics are reduced to find a solution of (3). Some methods have been proposed to solve the equilibrium problem (see [BO, FA, K1, K2, K3, KK, MT]).

Let $A: H \rightarrow H$ be a mapping. Then $A$ is called:

(1) monotone if

$$
\langle A x-A y, x-y\rangle \geq 0 \quad \forall x, y \in H
$$

(2) $\sigma$-strongly monotone if there exists a positive real number $\sigma$ such that

$$
\langle A x-A y, x-y\rangle \geq \sigma\|x-y\|^{2} \quad \forall x, y \in H .
$$

For constant $\sigma>0$, this implies that

$$
\|A x-A y\| \geq \sigma\|x-y\|,
$$

that is, $A$ is $\sigma$-expansive and when $\sigma=1$, it is expansive;

(3) $\sigma$-inverse-strongly monotone if there exists a positive real number $\sigma$ such that

$$
\langle A x-A y, x-y\rangle \geq \sigma\|A x-A y\|^{2} \quad \forall x, y \in H
$$

(4) $k$-strictly pseudo-contractive, if there exists a constant $k \in[0,1)$ such that

$$
\|A x-A y\|^{2} \leq\|x-y\|^{2}+k\|(I-A) x-(I-A) y\|^{2} \quad \forall x, y \in H .
$$


Let $A: H \rightarrow H$ be a single-valued nonlinear mapping and $M: H \rightarrow 2^{H}$ be a set-valued mapping. We consider the following variational inclusion problem, which is to find a point $u \in H$ such that

$$
\theta \in A(u)+M(u)
$$

where $\theta$ is the zero vector in $H$. The set of solutions of problem (4) is denoted by $I(A, M)$.

If $M=\partial \delta_{C}$, where $C$ is a nonempty closed convex subset of $H$ and $\delta_{C}: H \rightarrow[0, \infty]$ is the indicator function of $C$, i.e., $\delta_{C}(x)=0$ for $x \in C$ and $\delta_{C}(x)=+\infty$ for $x \notin C$, then the variational inclusion problem (4) is equivalent to finding $u \in C$ such that

$$
\langle A u, v-u\rangle \geq 0 \quad \forall v \in H .
$$

This problem is called the Hartman-Stampacchia variational problem ([Bro, HS, LS]). The set of solutions of problem (5) is denoted by $V I(C, A)$.

A set-valued mapping $M: H \rightarrow 2^{H}$ is called monotone if for all $x, y \in H, f \in M x$ and $g \in M y$ imply $\langle x-y, f-g\rangle \geq 0$. A monotone mapping $M: H \rightarrow 2^{H}$ is maximal if the graph of $G(M)$ of $M$ is not properly contained in the graph of any other monotone mapping. It is known that a monotone mapping $M$ is maximal if and only if for $(x, f) \in$ $H \times H,\langle x-y, f-g\rangle \geq 0$ for every $(y, g) \in G(M)$ implies $f \in M x$.

Let the set-valued mapping $M: H \rightarrow 2^{H}$ be maximal monotone. We define the resolvent operator $J_{M, \lambda}$ associated with $M$ and $\lambda$ as follows:

$$
J_{M, \lambda}(u)=(I+\lambda M)^{-1}(u) \quad u \in H,
$$

where $\lambda$ is a positive number. It is worth mentioning that the resolvent operator $J_{M, \lambda}$ is single-valued, nonexpansive and 1-inverse strongly monotone ([Bré, PWSY, PS, ZLC]).

In this paper, we introduce a new iterative process (16) below for finding a common element of the set of solutions for mixed equilibrium problems, the set of solutions of the variational inclusion problems for inverse strongly monotone mappings and the set of common fixed points for an infinite family of strictly pseudo-contractive mappings in a Hilbert space. Then, we prove strong convergence theorems which extend and improve the corresponding results of Peng and Yao [PY] and Plubtieng and Sriprad [PS].

2. Preliminaries. Let $H$ be a real Hilbert space with norm $\|\cdot\|$ and inner product $\langle\cdot, \cdot\rangle$ and let $C$ be a closed convex subset of $H$. Then

$$
\|x-y\|^{2}=\|x\|^{2}-\|y\|^{2}-2\langle x-y, y\rangle
$$

and

$$
\|\lambda x+(1-\lambda) y\|^{2}=\lambda\|x\|^{2}+(1-\lambda)\|y\|^{2}-\lambda(1-\lambda)\|x-y\|^{2}
$$

for all $x, y \in H$ and $\lambda \in \mathbf{R}$. For every point $x \in H$, there exists a unique nearest point in $C$, denoted by $P_{C} x$, such that

$$
\left\|x-P_{C} x\right\| \leq\|x-y\| \text { for all } y \in C .
$$

$P_{C}$ is called the metric projection of $H$ onto $C$. It is well known that $P_{C}$ is a nonexpansive mapping of $H$ onto $C$ and satisfies

$$
\left\langle x-y, P_{C} x-P_{C} y\right\rangle \geq\left\|P_{C} x-P_{C} y\right\|^{2}
$$


for every $x, y \in H$. Moreover, $P_{C} x$ is characterized by the following properties: $P_{C} x \in C$ and

$$
\begin{gathered}
\left\langle x-P_{C} x, y-P_{C} x\right\rangle \leq 0 \\
\|x-y\|^{2} \geq\left\|x-P_{C} x\right\|^{2}+\left\|y-P_{C} x\right\|^{2}
\end{gathered}
$$

for all $x \in H, y \in C$.

For solving the mixed equilibrium problem, let us give the following assumptions for the bifunction $F, \varphi$ and the set $C$ :

(A1) $F(x, x)=0$ for all $x \in C$;

(A2) $F$ is monotone, i.e., $F(x, y)+F(y, x) \leq 0$ for all $x, y \in C$;

(A3) for each $x, y, z \in C, \lim _{t \rightarrow 0} F(t z+(1-t) x, y) \leq F(x, y)$;

(A4) for each $x \in C, y \mapsto F(x, y)$ is convex and lower semicontinuous;

(A5) for each $y \in C, x \mapsto F(x, y)$ is weakly upper semicontinuous;

(B1) for each $x \in H$ and $r>0$, there exist a bounded subset $D_{x} \subseteq C$ and $y_{x} \in C$ such that for any $z \in C \backslash D_{x}$,

$$
F\left(z, y_{x}\right)+\varphi\left(y_{x}\right)+\frac{1}{r}\left\langle y_{x}-z, z-x\right\rangle<\varphi(z) ;
$$

(B2) $C$ is a bounded set.

Lemma 2.1 (Peng and Yao [PY]). Let $C$ be a nonempty closed convex subset of $H$. Let $F: C \times C \rightarrow \mathbf{R}$ be a bifunction satisfying (A1)-(A5) and let $\varphi: C \rightarrow \mathbf{R} \cup\{+\infty\}$ be a proper lower semicontinuous and convex function. Assume that either (B1) or (B2) holds. For $r>0$ and $x \in H$, define a mapping $T_{r}: H \rightarrow C$ as follows:

$$
T_{r}(x)=\left\{z \in C: F(z, y)+\varphi(y)+\frac{1}{r}\langle y-z, z-x\rangle \geq \varphi(z) \quad \forall y \in C\right\}
$$

for all $z \in H$. Then

1. For each $x \in H, T_{r}(x) \neq \emptyset$;

2. $T_{r}$ is single-valued;

3. $T_{r}$ is firmly nonexpansive, i.e., for any $x, y \in H,\left\|T_{r} x-T_{r} y\right\|^{2} \leq\left\langle T_{r} x-T_{r} y, x-y\right\rangle$;

4. $F\left(T_{r}\right)=\operatorname{MEP}(F, \varphi)$;

5. $\operatorname{MEP}(F, \varphi)$ is closed and convex.

In order to prove our main results, we need the following lemmas.

LEMma 2.2 (Zhou [Z]). If $V: C \rightarrow H$ is a $k$-strict pseudo-contraction, then

(1) the fixed point set $F(V)$ of $V$ is closed convex, so that the projection $P_{F(V)}$ is well defined;

(2) define a mapping $T: C \rightarrow H$ by

$$
T x=t x+(1-t) V x \quad \forall x \in C .
$$

If $t \in[k, 1)$, then $T$ is a nonexpansive mapping such that $F(V)=F(T)$.

A family of mappings $\left\{V_{i}: C \rightarrow H\right\}_{i=1}^{\infty}$ is called a family of uniformly $k$-strict pseudocontractions, if there exists a constant $k \in[0,1)$ such that

$$
\left\|V_{i} x-V_{i} y\right\|^{2} \leq\|x-y\|^{2}+k\left\|\left(I-V_{i}\right) x-\left(I-V_{i}\right) y\right\|^{2} \quad \forall x, y \in C \forall i \geq 1 .
$$


Let $\left\{V_{i}: C \rightarrow C\right\}_{i=1}^{\infty}$ be a countable family of uniformly $k$-strict pseudo-contractions. Let $\left\{T_{i}: C \rightarrow C\right\}_{i=1}^{\infty}$ be the sequence of nonexpansive mappings defined by (12), i.e.,

$$
T_{i} x=t x+(1-t) V_{i} x \quad \forall x \in C \forall i \geq 1, t \in[k, 1) .
$$

Let $\left\{T_{i}\right\}$ be a sequence of nonexpansive mappings of $C$ into itself defined by (13) and let $\left\{\mu_{i}\right\}$ be a sequence of nonnegative numbers in $[0,1]$. For each $n \geq 1$, define a mapping $W_{n}$ of $C$ into itself as follows:

$$
\begin{aligned}
U_{n, n+1} & =I, \\
U_{n, k} & =\mu_{k} T_{k} U_{n, k+1}+\left(1-\mu_{k}\right) I, \quad k=n, n-1, \ldots, 2, \\
W_{n}=U_{n, 1} & =\mu_{1} T_{1} U_{n, 2}+\left(1-\mu_{1}\right) I .
\end{aligned}
$$

Such a mapping $W_{n}$ is nonexpansive from $C$ to $C$ and it is called the $W$-mapping generated by $T_{1}, T_{2}, \ldots, T_{n}$ and $\mu_{1}, \mu_{2}, \ldots, \mu_{n}$.

Let for each $n, k \in \mathbf{N}$ the mapping $U_{n, k}$ be defined by (14). Then we can have the following crucial conclusions concerning $W_{n}$ which can be found in $[\mathrm{ST}]$. Now we only need the following similar version in Hilbert spaces.

Lemma 2.3 (Shimoji and Takahashi [ST]). Let $C$ be a nonempty closed convex subset of a real Hilbert space $H$. Let $T_{1}, T_{2}, \ldots$ be nonexpansive mappings of $C$ into itself such that $\bigcap_{n=1}^{\infty} F\left(T_{n}\right)$ is nonempty, let $\mu_{1}, \mu_{2}, \ldots$ be real numbers such that $0 \leq \mu_{n} \leq b<1$ for every $n \geq 1$. Then

(1) $W_{n}$ is nonexpansive and $F\left(W_{n}\right)=\bigcap_{i=1}^{n} F\left(T_{i}\right)$ for every $n \geq 1$;

(2) for every $x \in C$ and $k \in \mathbf{N}$, the limit $\lim _{n \rightarrow \infty} U_{n, k} x$ exists;

(3) a mapping $W: C \rightarrow C$ defined by

$$
W x:=\lim _{n \rightarrow \infty} W_{n} x=\lim _{n \rightarrow \infty} U_{n, 1} x \quad \forall x \in C
$$

is a nonexpansive mapping satisfying $F(W)=\bigcap_{i=1}^{\infty} F\left(T_{i}\right)$ and it is called the $W$ mapping generated by $T_{1}, T_{2}, \ldots$ and $\mu_{1}, \mu_{2}, \ldots$.

Lemma 2.4 (Chang $[\mathrm{C}]$ ). Let $C$ be a nonempty closed convex subset of a Hilbert space $H$, $\left\{T_{i}: C \rightarrow C\right\}$ be a countable family of nonexpansive mappings with $\bigcap_{i=1}^{\infty} F\left(T_{i}\right) \neq \emptyset,\left\{\mu_{i}\right\}$ be a real sequence such that $0<\mu_{i} \leq b<1$ for $i \geq 1$. If $D$ is any bounded subset of $C$, then

$$
\lim _{n \rightarrow \infty} \sup _{x \in D}\left\|W x-W_{n} x\right\|=0 .
$$

Lemma $2.5(\mathrm{Xu}[\mathrm{X}])$. Assume $\left\{a_{n}\right\}$ is a sequence of nonnegative real numbers such that

$$
a_{n+1} \leq\left(1-\alpha_{n}\right) a_{n}+\delta_{n}, n \geq 0,
$$

where $\left\{\alpha_{n}\right\}$ is a sequence in $(0,1)$ and $\left\{\delta_{n}\right\}$ is a sequence in $\mathbf{R}$ such that

(1) $\sum_{n=1}^{\infty} \alpha_{n}=\infty$,

(2) $\limsup _{n \rightarrow \infty} \frac{\delta_{n}}{\alpha_{n}} \leq 0$ or $\sum_{n=1}^{\infty}\left|\delta_{n}\right|<\infty$.

Then $\lim _{n \rightarrow \infty} a_{n}=0$. 
Lemma 2.6 (Osilike and Igbokwe $[\mathrm{OI}])$. Let $(E,\langle.,\rangle$.$) be an inner product space. Then$ for all $x, y, z \in E$ and $\alpha, \beta, \gamma \in[0,1]$ with $\alpha+\beta+\gamma=1$, we have

$$
\|\alpha x+\beta y+\gamma z\|^{2}=\alpha\|x\|^{2}+\beta\|y\|^{2}+\gamma\|z\|^{2}-\alpha \beta\|x-y\|^{2}-\alpha \gamma\|x-z\|^{2}-\beta \gamma\|y-z\|^{2} .
$$

Lemma 2.7 (Suzuki $[\mathrm{S}]$ ). Let $\left\{x_{n}\right\}$ and $\left\{y_{n}\right\}$ be bounded sequences in a Banach space $X$ and let $\left\{\beta_{n}\right\}$ be a sequence in $[0,1]$ with $0<\liminf _{n \rightarrow \infty} \beta_{n} \leq \limsup _{n \rightarrow \infty} \beta_{n}<1$. Suppose $x_{n+1}=\left(1-\beta_{n}\right) y_{n}+\beta_{n} x_{n}$ for all integers $n \geq 0$ and $\lim _{\sup _{n \rightarrow \infty}}\left(\left\|y_{n+1}-y_{n}\right\|-\right.$ $\left.\left\|x_{n+1}-x_{n}\right\|\right) \leq 0$. Then, $\lim _{n \rightarrow \infty}\left\|y_{n}-x_{n}\right\|=0$.

Lemma 2.8 (Marino and $\mathrm{Xu}[\mathrm{MX}]$ ). Assume $B$ is a strongly positive linear bounded operator on a Hilbert space $H$ with coefficient $\bar{\gamma}>0$ and $0<\rho \leq\|B\|^{-1}$. Then $\|I-\rho B\| \leq$ $1-\rho \bar{\gamma}$.

Lemma 2.9 (Opial [O]). Each Hilbert space H satisfies Opial's condition, i.e., for any sequence $\left\{x_{n}\right\} \subset H$ with $x_{n} \rightarrow x$, the inequality

$$
\liminf _{n \rightarrow \infty}\left\|x_{n}-x\right\|<\liminf _{n \rightarrow \infty}\left\|x_{n}-y\right\|,
$$

holds for each $y \in H$ with $y \neq x$.

Lemma 2.10 (Brézis [Bré]). Let $M: H \rightarrow 2^{H}$ be a maximal monotone mapping and $A: H \rightarrow H$ be a Lipschitz continuous mapping. Then the mapping $S=M+A: H \rightarrow 2^{H}$ is a maximal monotone mapping.

REMARK 2.11. Lemma 2.10 implies that $I(A, M)$ is closed and convex if $M: H \rightarrow 2^{H}$ is a maximal monotone mapping and $A: H \rightarrow H$ is a Lipschitz continuous mapping.

LEMMA 2.12 (Zhang et al. [ZLC]). $u \in H$ is a solution of variational inclusion (4) if and only if $u=J_{M, \lambda}(u-\lambda A u)$ for each $\lambda>0$, i.e.,

$$
I(A, M)=F\left(J_{M, \lambda}(I-\lambda A)\right) \quad \forall \lambda>0 .
$$

3. Main results. In this section, we show a strong convergence theorem for finding a common element of the set of solutions for mixed equilibrium problems, the set of solutions of the variational inclusion problems for inverse strongly monotone mappings and the set of common fixed points for an infinite family of strictly pseudo-contractive mappings in a Hilbert space by using the viscosity approximation method.

TheOREm 3.1. Let $C$ be a nonempty closed convex subset of a real Hilbert space H. Let $F$ be a bifunction of $C \times C$ into real numbers $\mathbf{R}$ satisfying (A1)-(A5) and let $\varphi: C \rightarrow$ $\mathbf{R} \cup\{+\infty\}$ be a proper lower semicontinuous and convex function. Let $f$ be a contraction of $H$ into itself with coefficient $\alpha \in(0,1)$ and $B$ be a strongly bounded linear operator on $H$ with coefficient $\bar{\gamma}>0$ and $0<\gamma<\frac{\bar{\gamma}}{\alpha}$. Let $M_{1}, M_{2}: H \rightarrow 2^{H}$ be maximal monotone mappings and $A_{1}, A_{2}: H \rightarrow H$ be $\sigma_{1}, \sigma_{2}$-inverse-strongly monotone mappings, respectively. Let $\left\{V_{i}: C \rightarrow C\right\}_{i=1}^{\infty}$ be a countable family of uniformly k-strict pseudocontractions, $\left\{T_{i}: C \rightarrow C\right\}_{i=1}^{\infty}$ be the countable family of nonexpansive mappings defined by $T_{i} x=t x+(1-t) V_{i} x$, for all $x \in C, i \geq 1, t \in[k, 1)$. Let $W_{n}$ be the $W$-mapping defined by (14) and $W$ be a mapping defined by (15) with $F(W) \neq \emptyset$. Assume that either (B1) or (B2) holds and $\Omega:=\bigcap_{n=1}^{N} F\left(T_{i}\right) \cap I\left(A_{1}, M_{1}\right) \cap I\left(A_{2}, M_{2}\right) \cap \operatorname{MEP}(F, \varphi) \neq \emptyset$. Let 
$\left\{x_{n}\right\},\left\{y_{n}\right\},\left\{z_{n}\right\}$ and $\left\{u_{n}\right\}$ be sequences generated by $x_{1} \in H$ and

$$
\left\{\begin{array}{l}
F\left(u_{n}, y\right)+\varphi(y)-\varphi\left(u_{n}\right)+\frac{1}{r_{n}}\left\langle y-u_{n}, u_{n}-x_{n}\right\rangle \geq 0 \quad \forall y \in C, \\
y_{n}=J_{M_{2}, \delta}\left(u_{n}-\delta A_{2} u_{n}\right) \\
v_{n}=J_{M_{1}, \tau}\left(y_{n}-\tau A_{1} y_{n}\right) \\
x_{n+1}=\alpha_{n} \gamma f\left(x_{n}\right)+\beta_{n} x_{n}+\left(\left(1-\beta_{n}\right) I-\alpha_{n} B\right) W_{n} v_{n}
\end{array}\right.
$$

for every $n \geq 1$, where $\left\{\alpha_{n}\right\},\left\{\beta_{n}\right\} \subset(0,1),\left\{r_{n}\right\} \subset(0, \infty), \tau \in\left(0,2 \sigma_{1}\right)$ and $\delta \in\left(0,2 \sigma_{2}\right)$ satisfy:

(i) $\sum_{n=0}^{\infty} \alpha_{n}=\infty$ and $\lim _{n \rightarrow \infty} \alpha_{n}=0$;

(ii) $\liminf _{n \rightarrow \infty} r_{n}>0$ and $\sum_{n=1}^{\infty}\left|r_{n+1}-r_{n}\right|<\infty$;

(iii) $0<\liminf _{n \rightarrow \infty} \beta_{n} \leq \limsup _{n \rightarrow \infty} \beta_{n}<1$.

Then $\left\{x_{n}\right\}$ converges strongly to $z \in \Omega$ which is the unique solution of the variational inequality

$$
\langle(B-\gamma f) z, z-x\rangle \leq 0, \quad x \in \Omega
$$

Equivalently, we have $z=P_{\Omega}(I-B+\gamma f)(z)$.

Proof. First, we show that $I-\tau A_{1}$ and $I-\delta A_{2}$ are nonexpansive. Indeed, for all $x, y \in H$ and $\tau \in\left(0,2 \sigma_{1}\right)$, we note that

$$
\begin{aligned}
\left\|\left(I-\tau A_{1}\right) u-\left(I-\tau A_{1}\right) v\right\|^{2} & =\left\|(u-v)-\tau\left(A_{1} u-A_{1} v\right)\right\|^{2} \\
& =\|u-v\|^{2}-2 \tau\left\langle u-v, A_{1} u-A_{1} v\right\rangle+\tau^{2}\left\|A_{1} u-A_{1} v\right\|^{2} \\
& \leq\|u-v\|^{2}+\tau\left(\tau-2 \sigma_{1}\right)\left\|A_{1} u-A_{1} v\right\|^{2} \leq\|u-v\|^{2}, \quad(17)
\end{aligned}
$$

which implies that the mapping $I-\tau A_{1}$ is nonexpansive. So is $I-\delta A_{2}$.

By condition (i), we may assume, without loss of generality, that $\alpha_{n}<\|B\|^{-1}$ for all $n$. We assume that $\|I-B\| \leq 1-\bar{\gamma}$. Since $B$ is a strongly positive bounded linear operator on $H$, we have

$$
\|B\|=\sup \{|\langle B x, x\rangle|: x \in H,\|x\|=1\} .
$$

Observe that

$$
\left\langle\left(\left(1-\beta_{n}\right) I-\alpha_{n} B\right) x, x\right\rangle=1-\beta_{n}-\alpha_{n}\langle B x, x\rangle \geq 1-\beta_{n}-\alpha_{n}\|B\| \geq 0,
$$

this shows that $\left(1-\beta_{n}\right) I-\alpha_{n} B$ is positive. It follows that

$$
\begin{aligned}
\left\|\left(1-\beta_{n}\right) I-\alpha_{n} B\right\| & =\sup \left\{\left|\left\langle\left(\left(1-\beta_{n}\right) I-\alpha_{n} B\right) x, x\right\rangle\right|: x \in H,\|x\|=1\right\} \\
& =\sup \left\{1-\beta_{n}-\alpha_{n}\langle B x, x\rangle: x \in H,\|x\|=1\right\} \\
& \leq 1-\beta_{n}-\alpha_{n} \bar{\gamma} .
\end{aligned}
$$

Let $p \in \Omega$, and let $\left\{T_{r_{n}}\right\}$ be a sequence of mappings defined as in Lemma 2.1 and $u_{n}=T_{r_{n}} x_{n}$. For any $n \in \mathbf{N}$, we have

$$
\left\|u_{n}-p\right\|=\left\|T_{r_{n}} x_{n}-T_{r_{n}} p\right\| \leq\left\|x_{n}-p\right\| .
$$

Since $p \in I\left(A_{1}, M_{1}\right)$ and $p \in I\left(A_{2}, M_{2}\right)$, we have $p=J_{M_{1}, \tau}\left(p-\tau A_{1} p\right)=J_{M_{2}, \delta}\left(p-\delta A_{2} p\right)$. 
Because $I-\tau A_{1}, I-\delta A_{2}, J_{M_{1}, \tau}$ and $J_{M_{2}, \delta}$ are nonexpansive mappings, we obtain

$$
\begin{aligned}
\left\|v_{n}-p\right\| & =\left\|J_{M_{1}, \tau}\left(y_{n}-\tau A_{1} z_{n}\right)-J_{M_{1}, \tau}\left(p-\tau A_{1} p\right)\right\| \\
& \leq\left\|\left(I-\tau A_{1}\right) y_{n}-\left(I-\tau A_{1}\right) p\right\| \leq\left\|y_{n}-p\right\| \\
& =\left\|J_{M_{2}, \delta}\left(u_{n}-\delta A_{2} u_{n}\right)-J_{M_{2}, \delta}\left(p-\delta A_{2} p\right)\right\| \\
& \leq\left\|\left(I-\delta A_{2}\right) u_{n}-\left(I-\delta A_{2}\right) p\right\| \\
& \leq\left\|u_{n}-p\right\| \leq\left\|x_{n}-p\right\| .
\end{aligned}
$$

It follows that

$$
\begin{aligned}
\left\|x_{n+1}-p\right\| & =\left\|\alpha_{n} \gamma f\left(x_{n}\right)+\beta_{n} x_{n}+\left(\left(1-\beta_{n}\right) I-\alpha_{n} B\right) W_{n} v_{n}-p\right\| \\
& =\left\|\alpha_{n}\left(\gamma f\left(x_{n}\right)-B p\right)+\beta_{n}\left(x_{n}-p\right)+\left(\left(1-\beta_{n}\right) I-\alpha_{n} B\right)\left(W_{n} v_{n}-p\right)\right\| \\
& \leq \alpha_{n}\left\|\gamma f\left(x_{n}\right)-B p\right\|+\beta_{n}\left\|x_{n}-p\right\|+\left(1-\beta_{n}-\alpha_{n} \bar{\gamma}\right)\left\|v_{n}-p\right\| \\
& \leq \alpha_{n}\left\|\gamma f\left(x_{n}\right)-B p\right\|+\beta_{n}\left\|x_{n}-p\right\|+\left(1-\beta_{n}-\alpha_{n} \bar{\gamma}\right)\left\|x_{n}-p\right\| \\
& \leq \alpha_{n}\left\|\gamma f\left(x_{n}\right)-\gamma f(p)\right\|+\alpha_{n}\|\gamma f(p)-B p\|+\left(1-\alpha_{n} \bar{\gamma}\right)\left\|x_{n}-p\right\| \\
& \leq \alpha_{n} \gamma \alpha\left\|x_{n}-p\right\|+\alpha_{n}\|\gamma f(p)-B p\|+\left(1-\alpha_{n} \bar{\gamma}\right)\left\|x_{n}-p\right\| \\
& =\left(1-(\bar{\gamma}-\gamma \alpha) \alpha_{n}\right)\left\|x_{n}-p\right\|+(\bar{\gamma}-\gamma \alpha) \alpha_{n} \frac{\|\gamma f(p)-B p\|}{\bar{\gamma}-\gamma \alpha}
\end{aligned}
$$

for every $n \in \mathbf{N}$. It follows by mathematical induction that

$$
\left\|x_{n+1}-p\right\| \leq \max \left\{\left\|x_{1}-p\right\|, \frac{\|\gamma f(p)-B p\|}{\bar{\gamma}-\gamma \alpha}\right\}, \quad n \geq 1 .
$$

Therefore $\left\{x_{n}\right\}$ is bounded, so $\left\{y_{n}\right\},\left\{u_{n}\right\},\left\{v_{n}\right\},\left\{f\left(x_{n}\right)\right\}$ and $\left\{W_{n} v_{n}\right\}$ are all bounded.

Next, we show that $\left\|x_{n+1}-x_{n}\right\| \rightarrow 0$ and $\left\|x_{n}-W_{n} v_{n}\right\| \rightarrow 0$ as $n \rightarrow 0$. Observing that $u_{n}=T_{r_{n}} x_{n} \in \operatorname{dom} \varphi$ and $u_{n+1}=T_{r_{n+1}} x_{n+1} \in \operatorname{dom} \varphi$ we get

$$
F\left(u_{n}, y\right)+\varphi(y)-\varphi\left(u_{n}\right)+\frac{1}{r_{n}}\left\langle y-u_{n}, u_{n}-x_{n}\right\rangle \geq 0 \text { for all } y \in C
$$

and

$$
F\left(u_{n+1}, y\right)+\varphi(y)-\varphi\left(u_{n+1}\right)+\frac{1}{r_{n+1}}\left\langle y-u_{n+1}, u_{n+1}-x_{n+1}\right\rangle \geq 0 \text { for all } y \in C .
$$

Take $y=u_{n+1}$ in (20) and $y=u_{n}$ in (21), by using condition (A2), we obtain

$$
\left\langle u_{n+1}-u_{n}, \frac{u_{n}-x_{n}}{r_{n}}-\frac{u_{n+1}-x_{n+1}}{r_{n+1}}\right\rangle \geq 0 .
$$

Thus $\left\langle u_{n+1}-u_{n}, u_{n}-u_{n+1}+x_{n+1}-x_{n}+\left(1-\frac{r_{n}}{r_{n+1}}\right)\left(u_{n+1}-x_{n+1}\right)\right\rangle \geq 0$. Without loss of generality, let us assume that there exists a real number $c$ such that $r_{n}>c$, for $n \geq 1$. Then we have

$$
\left\|u_{n+1}-u_{n}\right\|^{2} \leq\left\|u_{n+1}-u_{n}\right\|\left\{\left\|x_{n+1}-x_{n}\right\|+\left|1-\frac{r_{n}}{r_{n+1}}\right|\left\|u_{n+1}-x_{n+1}\right\|\right\}
$$

and hence

$$
\begin{aligned}
\left\|u_{n+1}-u_{n}\right\| & \leq\left\|x_{n+1}-x_{n}\right\|+\frac{1}{r_{n+1}}\left|r_{n+1}-r_{n}\right|\left\|u_{n+1}-x_{n+1}\right\| \\
& \leq\left\|x_{n+1}-x_{n}\right\|+\frac{1}{c}\left|r_{n+1}-r_{n}\right| M_{1},
\end{aligned}
$$


where $M_{1}=\sup \left\{\left\|u_{n}-x_{n}\right\|: n \in \mathbf{N}\right\}$. On the other hand, again since $I-\tau A_{1}, I-\delta A_{2}$, $J_{M_{1}, \tau}$ and $J_{M_{2}, \delta}$ are nonexpansive, we obtain

$$
\begin{aligned}
\left\|v_{n+1}-v_{n}\right\| & =\left\|J_{M_{1}, \tau}\left(y_{n+1}-\tau A_{1} y_{n+1}\right)-J_{M_{1}, \tau}\left(y_{n}-\tau A_{1} y_{n}\right)\right\| \\
& \leq\left\|\left(y_{n+1}-\tau A_{1} y_{n+1}\right)-\left(y_{n}-\tau A_{1} y_{n}\right)\right\| \leq\left\|y_{n+1}-y_{n}\right\| \\
& =\left\|J_{M_{2}, \delta}\left(u_{n+1}-\delta A_{2} u_{n+1}\right)-J_{M_{2}, \delta}\left(u_{n}-\delta A_{2} u_{n}\right)\right\| \\
& \leq\left\|\left(u_{n+1}-\delta A_{2} u_{n+1}\right)-\left(u_{n}-\delta B_{2} u_{n}\right)\right\| \leq\left\|u_{n+1}-u_{n}\right\| \\
& \leq\left\|x_{n+1}-x_{n}\right\|+\frac{1}{c}\left|r_{n+1}-r_{n}\right| M_{1} .
\end{aligned}
$$

Since $T_{i}$ and $U_{n, i}$ are nonexpansive, we have

$$
\begin{aligned}
\left\|W_{n+1} v_{n}-W_{n} v_{n}\right\| & =\left\|\mu_{1} T_{1} U_{n+1,2} v_{n}-\mu_{1} T_{1} U_{n, 2} v_{n}\right\| \\
& \leq \mu_{1}\left\|U_{n+1,2} v_{n}-U_{n, 2} v_{n}\right\| \\
& =\mu_{1}\left\|\mu_{2} T_{2} U_{n+1,3} v_{n}-\mu_{2} T_{2} U_{n, 3} v_{n}\right\| \\
& \leq \mu_{1} \mu_{2}\left\|U_{n+1,3} v_{n}-U_{n, 3} v_{n}\right\| \\
& \vdots \\
& \leq \mu_{1} \mu_{2} \cdots \mu_{n}\left\|U_{n+1, n+1} v_{n}-U_{n, n+1} v_{n}\right\| \\
& \leq M_{2} \prod_{i=1}^{n} \mu_{i},
\end{aligned}
$$

where $M_{2} \geq 0$ is a constant such that $\left\|U_{n+1, n+1} v_{n}-U_{n, n+1} v_{n}\right\| \leq M_{2}$ for all $n \geq 0$. It follows from (23) and (24) that

$$
\begin{aligned}
\left\|W_{n+1} v_{n+1}-W_{n} v_{n}\right\| & \leq\left\|W_{n+1} v_{n+1}-W_{n+1} v_{n}\right\|+\left\|W_{n+1} v_{n}-W_{n} v_{n}\right\| \\
& \leq\left\|v_{n+1}-v_{n}\right\|+M_{2} \prod_{i=1}^{n} \mu_{i} \\
& \leq\left\|x_{n+1}-x_{n}\right\|+\frac{1}{c}\left|r_{n+1}-r_{n}\right| M_{1}+M_{2} \prod_{i=1}^{n} \mu_{i} .
\end{aligned}
$$

Define the sequence $\left\{z_{n}\right\}$ by $x_{n+1}=\left(1-\beta_{n}\right) z_{n}+\beta_{n} x_{n}$, for each $n \geq 1$. Then, observe that

$$
\begin{aligned}
z_{n} & =\frac{x_{n+1}-\beta_{n} x_{n}}{1-\beta_{n}} \\
& =\frac{\alpha_{n} \gamma f\left(x_{n}\right)+\beta_{n} x_{n}+\left(\left(1-\beta_{n}\right) I-\alpha_{n} B\right) W_{n} v_{n}-\beta_{n} x_{n}}{1-\beta_{n}} \\
& =\frac{\alpha_{n} \gamma f\left(x_{n}\right)+\left(\left(1-\beta_{n}\right) I-\alpha_{n} B\right) W_{n} v_{n}}{1-\beta_{n}}
\end{aligned}
$$

and hence

$$
\begin{gathered}
z_{n+1}-z_{n}=\frac{\alpha_{n+1} \gamma f\left(x_{n+1}\right)+\left(\left(1-\beta_{n+1}\right) I-\alpha_{n+1} B\right) W_{n+1} v_{n+1}}{1-\beta_{n+1}} \\
-\frac{\alpha_{n} \gamma f\left(x_{n}\right)+\left(\left(1-\beta_{n}\right) I-\alpha_{n} B\right) W_{n} v_{n}}{1-\beta_{n}}
\end{gathered}
$$




$$
\begin{aligned}
& =\frac{\alpha_{n+1} \gamma f\left(x_{n+1}\right)}{1-\beta_{n+1}}+\frac{\left(1-\beta_{n+1}\right) W_{n+1} v_{n+1}}{1-\beta_{n+1}}-\frac{\alpha_{n+1} B W_{n+1} v_{n+1}}{1-\beta_{n+1}} \\
& \quad-\frac{\alpha_{n} \gamma f\left(x_{n}\right)}{1-\beta_{n}}-\frac{\left(1-\beta_{n}\right) W_{n} v_{n}}{1-\beta_{n}}+\frac{\alpha_{n} B W_{n} v_{n}}{1-\beta_{n}} \\
& =\frac{\alpha_{n+1}}{1-\beta_{n+1}}\left(\gamma f\left(x_{n+1}\right)-B W_{n+1} v_{n+1}\right)+\frac{\alpha_{n}}{1-\beta_{n}}\left(B W_{n} v_{n}-\gamma f\left(x_{n}\right)\right) \\
& \quad+W_{n+1} v_{n+1}-W_{n} v_{n} .
\end{aligned}
$$

Combining this with (25), we obtain

$$
\begin{aligned}
\left\|z_{n+1}-z_{n}\right\| \leq & \frac{\alpha_{n+1}}{1-\beta_{n+1}}\left(\left\|\gamma f\left(x_{n+1}\right)\right\|+\left\|B W_{n+1} v_{n+1}\right\|\right) \\
& +\frac{\alpha_{n}}{1-\beta_{n}}\left(\left\|B W_{n} v_{n}\right\|+\left\|\gamma f\left(x_{n}\right)\right\|\right)+\left\|W_{n+1} v_{n+1}-W_{n} v_{n}\right\| \\
\leq & \frac{\alpha_{n+1}}{1-\beta_{n+1}}\left(\left\|\gamma f\left(x_{n+1}\right)\right\|+\left\|B W_{n+1} v_{n+1}\right\|\right) \\
& +\frac{\alpha_{n}}{1-\beta_{n}}\left(\left\|B W_{n} v_{n}\right\|+\left\|\gamma f\left(x_{n}\right)\right\|\right) \\
& +\left\|x_{n+1}-x_{n}\right\|+\frac{1}{c}\left|r_{n+1}-r_{n}\right| M_{1}+M_{2} \prod_{i=1}^{n} \mu_{i} .
\end{aligned}
$$

Observe that

$$
\begin{aligned}
&\left\|z_{n+1}-z_{n}\right\|-\left\|x_{n+1}-x_{n}\right\| \leq \frac{\alpha_{n+1}}{1-\beta_{n+1}}\left(\left\|\gamma f\left(x_{n+1}\right)\right\|+\left\|B W_{n+1} v_{n+1}\right\|\right) \\
&+\frac{\alpha_{n}}{1-\beta_{n}}\left(\left\|B W_{n} v_{n}\right\|+\left\|\gamma f\left(x_{n}\right)\right\|\right)+\frac{1}{c}\left|r_{n+1}-r_{n}\right| M_{1}+M_{2} \prod_{i=1}^{n} \mu_{i} .
\end{aligned}
$$

Assumptions (i)-(iii) imply that

$$
\limsup _{n \rightarrow \infty}\left(\left\|z_{n+1}-z_{n}\right\|-\left\|x_{n+1}-x_{n}\right\|\right) \leq 0 .
$$

Hence, by Lemma 2.7, we have

$$
\lim _{n \rightarrow \infty}\left\|z_{n}-x_{n}\right\|=0
$$

Consequently,

$$
\lim _{n \rightarrow \infty}\left\|x_{n+1}-x_{n}\right\|=\lim _{n \rightarrow \infty}\left(1-\beta_{n}\right)\left\|z_{n}-x_{n}\right\|=0 .
$$

From (ii), (23) and (27), we have $\left\|u_{n+1}-u_{n}\right\| \rightarrow 0,\left\|y_{n+1}-y_{n}\right\| \rightarrow 0$ and $\left\|v_{n+1}-v_{n}\right\| \rightarrow 0$ as $n \rightarrow \infty$. We note that

$$
\begin{aligned}
x_{n+1}-x_{n}= & \alpha_{n} \gamma f\left(x_{n}\right)+\beta_{n} x_{n}+\left(\left(1-\beta_{n}\right) I-\alpha_{n} B\right) W_{n} v_{n}-x_{n} \\
= & \alpha_{n} \gamma f\left(x_{n}\right)-\alpha_{n} B x_{n}+\alpha_{n} B x_{n}+\beta_{n} x_{n}+\left(\left(1-\beta_{n}\right) I-\alpha_{n} B\right) W_{n} v_{n} \\
& \quad-\left(\left(1-\beta_{n}\right) I-\alpha_{n} B\right) x_{n}+\left(\left(1-\beta_{n}\right) I-\alpha_{n} B\right) x_{n}-x_{n} \\
= & \alpha_{n}\left(\gamma f\left(x_{n}\right)-B x_{n}\right)+\left(\left(1-\beta_{n}\right) I-\alpha_{n} B\right)\left(W_{n} v_{n}-x_{n}\right),
\end{aligned}
$$

hence

$$
\left(1-\beta_{n}-\alpha_{n} \bar{\gamma}\right)\left\|x_{n}-W_{n} v_{n}\right\| \leq \alpha_{n}\left\|\gamma f\left(x_{n}\right)-B x_{n}\right\|+\left\|x_{n}-x_{n+1}\right\| .
$$


From (i)-(iii) and (27) we obtain

$$
\lim _{n \rightarrow \infty}\left\|W_{n} v_{n}-x_{n}\right\|=0 .
$$

Next, we shall show that $\lim _{n \rightarrow \infty}\left\|u_{n}-x_{n}\right\|=0$. For any $p \in \Omega$ and $T_{r_{n}}$ is firmly nonexpansive, and we have

$$
\begin{aligned}
\left\|u_{n}-p\right\|^{2} & =\left\|T_{r_{n}} x_{n}-T_{r_{n}} p\right\|^{2} \leq\left\langle T_{r_{n}} x_{n}-T_{r_{n}} p, x_{n}-p\right\rangle=\left\langle u_{n}-p, x_{n}-p\right\rangle \\
& =\frac{1}{2}\left(\left\|u_{n}-p\right\|^{2}+\left\|x_{n}-p\right\|^{2}-\left\|u_{n}-x_{n}\right\|^{2}\right) .
\end{aligned}
$$

It follows that

$$
\left\|u_{n}-p\right\|^{2} \leq\left\|x_{n}-p\right\|^{2}-\left\|u_{n}-x_{n}\right\|^{2}
$$

Therefore, we have

$$
\begin{aligned}
\left\|x_{n+1}-p\right\|^{2} \leq & \alpha_{n}\left\|\gamma f\left(x_{n}\right)-B p\right\|^{2}+\beta_{n}\left\|x_{n}-p\right\|^{2}+\left(1-\beta_{n}-\alpha_{n} \bar{\gamma}\right)\left\|W_{n} v_{n}-p\right\|^{2} \\
\leq & \alpha_{n}\left\|\gamma f\left(x_{n}\right)-B p\right\|^{2}+\beta_{n}\left\|x_{n}-p\right\|^{2}+\left(1-\beta_{n}-\alpha_{n} \bar{\gamma}\right)\left\|v_{n}-p\right\|^{2} \\
\leq & \alpha_{n}\left\|\gamma f\left(x_{n}\right)-B p\right\|^{2}+\beta_{n}\left\|x_{n}-p\right\|^{2}+\left(1-\beta_{n}-\alpha_{n} \bar{\gamma}\right)\left\|u_{n}-p\right\|^{2} \\
\leq & \alpha_{n}\left\|\gamma f\left(x_{n}\right)-B p\right\|^{2}+\beta_{n}\left\|x_{n}-p\right\|^{2} \\
& \quad+\left(1-\beta_{n}-\alpha_{n} \bar{\gamma}\right)\left(\left\|x_{n}-p\right\|^{2}-\left\|u_{n}-x_{n}\right\|^{2}\right) \\
& =\alpha_{n}\left\|\gamma f\left(x_{n}\right)-B p\right\|^{2}+\left(1-\alpha_{n} \bar{\gamma}\right)\left\|x_{n}-p\right\|^{2} \\
& \quad-\left(1-\beta_{n}-\alpha_{n} \bar{\gamma}\right)\left\|u_{n}-x_{n}\right\|^{2} .
\end{aligned}
$$

It follows that

$$
\begin{array}{r}
\left(1-\beta_{n}-\alpha_{n} \bar{\gamma}\right)\left\|u_{n}-x_{n}\right\|^{2} \leq \alpha_{n}\left\|\gamma f\left(x_{n}\right)-B p\right\|^{2}+\left(1-\alpha_{n} \bar{\gamma}\right)\left\|x_{n}-p\right\|^{2}-\left\|x_{n+1}-p\right\|^{2} \\
\leq \alpha_{n}\left\|\gamma f\left(x_{n}\right)-B p\right\|^{2}+\left\|x_{n+1}-x_{n}\right\|\left(\left\|x_{n}-p\right\|+\left\|x_{n+1}-p\right\|\right) .
\end{array}
$$

Assumptions (i)-(iii) and formula (27) imply that

$$
\lim _{n \rightarrow \infty}\left\|x_{n}-u_{n}\right\|=0
$$

and by (ii) we have

$$
\lim _{n \rightarrow \infty}\left\|\frac{x_{n}-u_{n}}{r_{n}}\right\|=\lim _{n \rightarrow \infty} \frac{1}{r_{n}}\left\|x_{n}-u_{n}\right\|=0 .
$$

We note that, by (29), nonexpansiveness of $J_{M_{1}, \tau}, J_{M_{2}, \delta}$ and the inverse-strong monotonicity of $A_{1}, A_{2}$ imply that

$$
\begin{aligned}
&\left\|x_{n+1}-p\right\|^{2} \leq \alpha_{n}\left\|\gamma f\left(x_{n}\right)-B p\right\|^{2}+\beta_{n}\left\|x_{n}-p\right\|^{2}+\left(1-\beta_{n}-\alpha_{n} \bar{\gamma}\right)\left\|v_{n}-p\right\|^{2} \\
&= \alpha_{n}\left\|\gamma f\left(x_{n}\right)-B p\right\|^{2}+\beta_{n}\left\|x_{n}-p\right\|^{2} \\
& \quad+\left(1-\beta_{n}-\alpha_{n} \bar{\gamma}\right)\left\|J_{M_{1}, \tau}\left(y_{n}-\tau A_{1} y_{n}\right)-J_{M_{1}, \tau}\left(p-\tau A_{1} p\right)\right\|^{2} \\
& \leq \alpha_{n}\left\|\gamma f\left(x_{n}\right)-B p\right\|^{2}+\beta_{n}\left\|x_{n}-p\right\|^{2} \\
& \quad+\left(1-\beta_{n}-\alpha_{n} \bar{\gamma}\right)\left\|\left(I-\tau A_{1}\right) y_{n}-\left(I-\tau A_{1}\right) p\right\|^{2} \\
& \leq \alpha_{n}\left\|\gamma f\left(x_{n}\right)-B p\right\|^{2}+\beta_{n}\left\|x_{n}-p\right\|^{2} \quad+\left(1-\beta_{n}-\alpha_{n} \bar{\gamma}\right)\left\{\left\|y_{n}-p\right\|^{2}+\tau\left(\tau-2 \sigma_{1}\right)\left\|A_{1} y_{n}-A_{1} p\right\|^{2}\right\} \\
& \leq \alpha_{n}\left\|\gamma f\left(x_{n}\right)-B p\right\|^{2}+\beta_{n}\left\|x_{n}-p\right\|^{2}+\left(1-\beta_{n}-\alpha_{n} \bar{\gamma}\right)\left\|x_{n}-p\right\|^{2} \\
& \quad+\left(1-\beta_{n}-\alpha_{n} \bar{\gamma}\right) \tau\left(\tau-2 \sigma_{1}\right)\left\|A_{1} y_{n}-A_{1} p\right\|^{2}
\end{aligned}
$$




$$
\begin{aligned}
\leq \alpha_{n}\left\|\gamma f\left(x_{n}\right)-B p\right\|^{2}+\left\|x_{n}-p\right\|^{2} \\
\quad+\left(1-\beta_{n}-\alpha_{n} \bar{\gamma}\right) \tau\left(\tau-2 \sigma_{1}\right)\left\|A_{1} y_{n}-A_{1} p\right\|^{2}
\end{aligned}
$$

and

$$
\begin{aligned}
\left\|x_{n+1}-p\right\|^{2} \leq & \alpha_{n}\left\|\gamma f\left(x_{n}\right)-B p\right\|^{2}+\beta_{n}\left\|x_{n}-p\right\|^{2}+\left(1-\beta_{n}-\alpha_{n} \bar{\gamma}\right)\left\|y_{n}-p\right\|^{2} \\
= & \alpha_{n}\left\|\gamma f\left(x_{n}\right)-B p\right\|^{2}+\beta_{n}\left\|x_{n}-p\right\|^{2} \\
& \quad+\left(1-\beta_{n}-\alpha_{n} \bar{\gamma}\right)\left\|J_{M_{2}, \delta}\left(u_{n}-\delta A_{2} u_{n}\right)-J_{M_{2}, \delta}\left(p-\delta A_{2} p\right)\right\|^{2} \\
\leq & \alpha_{n}\left\|\gamma f\left(x_{n}\right)-B p\right\|^{2}+\beta_{n}\left\|x_{n}-p\right\|^{2} \\
& \quad+\left(1-\beta_{n}-\alpha_{n} \bar{\gamma}\right)\left\|\left(I-\delta A_{2}\right) u_{n}-\left(I-\delta A_{2}\right) p\right\|^{2} \\
\leq & \alpha_{n}\left\|\gamma f\left(x_{n}\right)-B p\right\|^{2}+\beta_{n}\left\|x_{n}-p\right\|^{2} \\
& \quad+\left(1-\beta_{n}-\alpha_{n} \bar{\gamma}\right)\left\{\left\|u_{n}-p\right\|^{2}+\delta\left(\delta-2 \sigma_{2}\right)\left\|A_{2} u_{n}-A_{2} p\right\|^{2}\right\} \\
\leq & \alpha_{n}\left\|\gamma f\left(x_{n}\right)-B p\right\|^{2}+\beta_{n}\left\|x_{n}-p\right\|^{2} \\
& \quad+\left(1-\beta_{n}-\alpha_{n} \bar{\gamma}\right)\left\|x_{n}-p\right\|^{2}+\left(1-\beta_{n}-\alpha_{n} \bar{\gamma}\right) \delta\left(\delta-2 \sigma_{2}\right)\left\|A_{2} u_{n}-A_{2} p\right\|^{2} \\
\leq & \alpha_{n}\left\|\gamma f\left(x_{n}\right)-B p\right\|^{2}+\left\|x_{n}-p\right\|^{2} \\
& \quad+\left(1-\beta_{n}-\alpha_{n} \bar{\gamma}\right) \delta\left(\delta-2 \sigma_{2}\right)\left\|A_{2} u_{n}-A_{2} p\right\|^{2}
\end{aligned}
$$

which imply that

$$
\begin{aligned}
0 & \leq\left(1-\beta_{n}-\alpha_{n} \bar{\gamma}\right) \tau\left(2 \sigma_{1}-\tau\right)\left\|A_{1} y_{n}-A_{1} p\right\|^{2} \\
& \leq \alpha_{n}\left\|\gamma f\left(x_{n}\right)-B p\right\|^{2}+\left\|x_{n}-p\right\|^{2}-\left\|x_{n+1}-p\right\|^{2} \\
& \leq \alpha_{n}\left\|\gamma f\left(x_{n}\right)-B p\right\|^{2}+\left\|x_{n}-x_{n+1}\right\|\left(\left\|x_{n}-p\right\|+\left\|x_{n+1}-p\right\|\right)
\end{aligned}
$$

and

$$
\begin{aligned}
0 & \leq\left(1-\beta_{n}-\alpha_{n} \bar{\gamma}\right) \delta\left(2 \sigma_{2}-\delta\right)\left\|A_{2} u_{n}-A_{2} p\right\|^{2} \\
& \leq \alpha_{n}\left\|\gamma f\left(x_{n}\right)-B p\right\|^{2}+\left\|x_{n}-p\right\|^{2}-\left\|x_{n+1}-p\right\|^{2} \\
& \leq \alpha_{n}\left\|\gamma f\left(x_{n}\right)-B p\right\|^{2}+\left\|x_{n}-x_{n+1}\right\|\left(\left\|x_{n}-p\right\|+\left\|x_{n+1}-p\right\|\right) .
\end{aligned}
$$

It follows from (i), (iii) and (27) that

$$
\lim _{n \rightarrow \infty}\left\|A_{1} y_{n}-A_{1} p\right\|=0
$$

and

$$
\lim _{n \rightarrow \infty}\left\|A_{2} u_{n}-A_{2} p\right\|=0 .
$$

On the other hand, since $J_{M_{1}, \tau}$ is firmly nonexpansive, we have

$$
\begin{aligned}
\left\|v_{n}-p\right\|^{2}= & \left\|J_{M_{1}, \tau}\left(y_{n}-\tau A_{1} y_{n}\right)-J_{M_{1}, \tau}\left(p-\tau A_{1} p\right)\right\|^{2} \\
\leq & \left\langle\left(y_{n}-\tau A_{1} y_{n}\right)-\left(p-\tau A_{1} p\right), v_{n}-p\right\rangle \\
= & \frac{1}{2}\left\{\left\|\left(y_{n}-\tau A_{1} y_{n}\right)-\left(p-\tau A_{1} p\right)\right\|^{2}+\left\|v_{n}-p\right\|^{2}\right. \\
& \left.\quad-\left\|\left(y_{n}-\tau A_{1} y_{n}\right)-\left(p-\tau A_{1} p\right)-\left(v_{n}-p\right)\right\|^{2}\right\} \\
\leq & \frac{1}{2}\left\{\left\|y_{n}-p\right\|^{2}+\left\|v_{n}-p\right\|^{2}-\left\|\left(y_{n}-v_{n}\right)-\tau\left(A_{1} y_{n}-A_{1} p\right)\right\|^{2}\right\}
\end{aligned}
$$




$$
\begin{aligned}
= & \frac{1}{2}\left\{\left\|y_{n}-p\right\|^{2}+\left\|v_{n}-p\right\|^{2}\right. \\
& \left.\quad-\left\|y_{n}-v_{n}\right\|^{2}+2 \tau\left\langle y_{n}-v_{n}, A_{1} y_{n}-A_{1} p\right\rangle-\tau^{2}\left\|A_{1} y_{n}-A_{1} p\right\|^{2}\right\} \\
\leq & \frac{1}{2}\left\{\left\|y_{n}-p\right\|^{2}+\left\|v_{n}-p\right\|^{2}\right. \\
& \left.\quad-\left\|y_{n}-v_{n}\right\|^{2}+2 \tau\left\|y_{n}-v_{n}\right\|\left\|A_{1} y_{n}-A_{1} p\right\|-\tau^{2}\left\|A_{1} y_{n}-A_{1} p\right\|^{2}\right\} \\
\leq & \frac{1}{2}\left\{\left\|x_{n}-p\right\|^{2}+\left\|v_{n}-p\right\|^{2}-\left\|y_{n}-v_{n}\right\|^{2}+2 \tau\left\|y_{n}-v_{n}\right\|\left\|A_{1} y_{n}-A_{1} p\right\|\right\},
\end{aligned}
$$

which yields that

$$
\left\|v_{n}-p\right\|^{2} \leq\left\|x_{n}-p\right\|^{2}-\left\|y_{n}-v_{n}\right\|^{2}+2 \tau\left\|y_{n}-v_{n}\right\|\left\|A_{1} y_{n}-A_{1} p\right\| .
$$

Similarly, since $J_{M_{2}, \delta}$ is firmly nonexpansive, we also have

$$
\left\|y_{n}-p\right\|^{2} \leq\left\|x_{n}-p\right\|^{2}-\left\|u_{n}-y_{n}\right\|^{2}+2 \delta\left\|u_{n}-y_{n}\right\|\left\|A_{2} u_{n}-A_{2} p\right\| .
$$

Substituting (33) into (29), we have

$$
\begin{aligned}
\left\|x_{n+1}-p\right\|^{2} \leq & \alpha_{n}\left\|\gamma f\left(x_{n}\right)-B p\right\|^{2}+\beta_{n}\left\|x_{n}-p\right\|^{2}+\left(1-\beta_{n}-\alpha_{n} \bar{\gamma}\right)\left\|W_{n} v_{n}-p\right\|^{2} \\
\leq & \alpha_{n}\left\|\gamma f\left(x_{n}\right)-B p\right\|^{2}+\beta_{n}\left\|x_{n}-p\right\|^{2}+\left(1-\beta_{n}-\alpha_{n} \bar{\gamma}\right)\left\|v_{n}-p\right\|^{2} \\
\leq & \alpha_{n}\left\|\gamma f\left(x_{n}\right)-B p\right\|^{2}+\beta_{n}\left\|x_{n}-p\right\|^{2} \\
& +\left(1-\beta_{n}-\alpha_{n} \bar{\gamma}\right)\left(\left\|x_{n}-p\right\|^{2}-\left\|y_{n}-v_{n}\right\|^{2}+2 \tau\left\|y_{n}-v_{n}\right\|\left\|A_{1} y_{n}-A_{1} p\right\|\right) \\
\leq & \alpha_{n}\left\|\gamma f\left(x_{n}\right)-B p\right\|^{2}+\left\|x_{n}-p\right\|^{2}-\left(1-\beta_{n}-\alpha_{n} \bar{\gamma}\right)\left\|y_{n}-v_{n}\right\|^{2} \\
& +2 \tau\left(1-\beta_{n}-\alpha_{n} \bar{\gamma}\right)\left\|y_{n}-v_{n}\right\|\left\|A_{1} y_{n}-A_{1} p\right\|
\end{aligned}
$$

and substituting (34) into (29), we get

$$
\begin{aligned}
\left\|x_{n+1}-p\right\|^{2} \leq & \alpha_{n}\left\|\gamma f\left(x_{n}\right)-B p\right\|^{2}+\beta_{n}\left\|x_{n}-p\right\|^{2}+\left(1-\beta_{n}-\alpha_{n} \bar{\gamma}\right)\left\|W_{n} v_{n}-p\right\|^{2} \\
\leq & \alpha_{n}\left\|\gamma f\left(x_{n}\right)-B p\right\|^{2}+\beta_{n}\left\|x_{n}-p\right\|^{2}+\left(1-\beta_{n}-\alpha_{n} \bar{\gamma}\right)\left\|v_{n}-p\right\|^{2} \\
\leq & \alpha_{n}\left\|\gamma f\left(x_{n}\right)-B p\right\|^{2}+\beta_{n}\left\|x_{n}-p\right\|^{2}+\left(1-\beta_{n}-\alpha_{n} \bar{\gamma}\right)\left\|y_{n}-p\right\|^{2} \\
\leq & \alpha_{n}\left\|\gamma f\left(x_{n}\right)-B p\right\|^{2}+\beta_{n}\left\|x_{n}-p\right\|^{2} \\
& +\left(1-\beta_{n}-\alpha_{n} \bar{\gamma}\right)\left(\left\|x_{n}-p\right\|^{2}-\left\|u_{n}-y_{n}\right\|^{2}+2 \delta\left\|u_{n}-y_{n}\right\|\left\|A_{2} u_{n}-A_{2} p\right\|\right) \\
\leq & \alpha_{n}\left\|\gamma f\left(x_{n}\right)-B p\right\|^{2}+\left\|x_{n}-p\right\|^{2}-\left(1-\beta_{n}-\alpha_{n} \bar{\gamma}\right)\left\|u_{n}-y_{n}\right\|^{2} \\
& +2 \delta\left(1-\beta_{n}-\alpha_{n} \bar{\gamma}\right)\left\|u_{n}-y_{n}\right\|\left\|A_{2} u_{n}-A_{2} p\right\| .
\end{aligned}
$$

Therefore, by (35) and (36), we have

$$
\begin{aligned}
&\left(1-\beta_{n}-\alpha_{n} \bar{\gamma}\right)\left\|y_{n}-v_{n}\right\|^{2} \leq \alpha_{n}\left\|\gamma f\left(x_{n}\right)-B p\right\|^{2}+\left\|x_{n}-p\right\|^{2}-\left\|x_{n+1}-p\right\|^{2} \\
&+2 \tau\left(1-\beta_{n}-\alpha_{n} \bar{\gamma}\right)\left\|y_{n}-v_{n}\right\|\left\|A_{1} y_{n}-A_{1} p\right\| \\
& \leq \alpha_{n}\left\|\gamma f\left(x_{n}\right)-B p\right\|^{2}+\left\|x_{n}-x_{n+1}\right\|\left(\left\|x_{n}-p\right\|+\left\|x_{n+1}-p\right\|\right) \\
&+2 \tau\left(1-\beta_{n}-\alpha_{n} \bar{\gamma}\right)\left\|y_{n}-v_{n}\right\|\left\|A_{1} y_{n}-A_{1} p\right\|
\end{aligned}
$$


and

$$
\begin{aligned}
&\left(1-\beta_{n}-\alpha_{n} \bar{\gamma}\right)\left\|u_{n}-y_{n}\right\|^{2} \leq \alpha_{n}\left\|\gamma f\left(x_{n}\right)-B p\right\|^{2}+\left\|x_{n}-p\right\|^{2}-\left\|x_{n+1}-p\right\|^{2} \\
&+2 \delta\left(1-\beta_{n}-\alpha_{n} \bar{\gamma}\right)\left\|u_{n}-y_{n}\right\|\left\|A_{2} u_{n}-A_{2} p\right\| \\
& \leq \alpha_{n}\left\|\gamma f\left(x_{n}\right)-B p\right\|^{2}+\left\|x_{n}-x_{n+1}\right\|\left(\left\|x_{n}-p\right\|+\left\|x_{n+1}-p\right\|\right) \\
&+
\end{aligned}
$$

It follows from (i), (iii) and (27) that

$$
\lim _{n \rightarrow \infty}\left\|y_{n}-v_{n}\right\|=0
$$

and

$$
\lim _{n \rightarrow \infty}\left\|u_{n}-y_{n}\right\|=0
$$

From (28), (30), (39) and (40), we have

$$
\left\|W_{n} v_{n}-v_{n}\right\| \leq\left\|W_{n} v_{n}-x_{n}\right\|+\left\|x_{n}-u_{n}\right\|+\left\|u_{n}-y_{n}\right\|+\left\|y_{n}-v_{n}\right\| \rightarrow 0 \quad \text { as } \quad n \rightarrow \infty
$$

and also

$$
\left\|v_{n}-x_{n}\right\| \leq\left\|v_{n}-y_{n}\right\|+\left\|y_{n}-u_{n}\right\|+\left\|u_{n}-x_{n}\right\| \rightarrow 0 \quad \text { as } n \rightarrow \infty .
$$

Observe that $P_{\Omega}(I-B+\gamma f)$ is a contraction of $H$ into itself. Indeed, for all $x, y \in H$, we have

$$
\begin{aligned}
\left\|P_{\Omega}(I-B+\gamma f)(x)-P_{\Omega}(I-B+\gamma f)(y)\right\| & \leq\|(I-B+\gamma f)(x)-(I-B+\gamma f)(y)\| \\
& \leq\|I-B\|\|x-y\|+\gamma\|f(x)-f(y)\| \\
& \leq(1-\bar{\gamma})\|x-y\|+\gamma \alpha\|x-y\| \\
& =(1-(\bar{\gamma}-\gamma \alpha))\|x-y\| .
\end{aligned}
$$

Since $H$ is complete, there exists a unique fixed point $z \in H$ such that

$$
z=P_{\Omega}(I-B+\gamma f)(z) .
$$

Next, we show that

$$
\limsup _{n \rightarrow \infty}\left\langle(B-\gamma f) z, z-x_{n}\right\rangle \leq 0 .
$$

Indeed, we can choose a subsequence $\left\{v_{n_{i}}\right\}$ of $\left\{v_{n}\right\}$ such that

$$
\lim _{i \rightarrow \infty}\left\langle(B-\gamma f) z, z-v_{n_{i}}\right\rangle=\limsup _{n \rightarrow \infty}\left\langle(B-\gamma f) z, z-v_{n}\right\rangle .
$$

Since $\left\{v_{n_{i}}\right\}$ is bounded, there exists a subsequence $\left\{v_{n_{i_{j}}}\right\}$ of $\left\{v_{n_{i}}\right\}$ which converges weakly to $v \in C$. Without loss of generality, we can assume that $v_{n_{i}} \rightarrow v$. From $\left\|W_{n} v_{n}-v_{n}\right\| \rightarrow 0$, we obtain $W_{n} v_{n_{i}} \rightarrow v$. Let us show that $v \in \operatorname{MEP}(F, \varphi)$. Since $u_{n}=T_{r_{n}} x_{n} \in \operatorname{dom} \varphi$, we have

$$
F\left(u_{n}, y\right)+\varphi(y)-\varphi\left(u_{n}\right)+\frac{1}{r_{n}}\left\langle y-u_{n}, u_{n}-x_{n}\right\rangle \geq 0 \quad \forall y \in C .
$$

From (A2) we also have

$$
\varphi(y)-\varphi\left(u_{n}\right)+\frac{1}{r_{n}}\left\langle y-u_{n}, u_{n}-x_{n}\right\rangle \geq F\left(y, u_{n}\right) \quad \forall y \in C,
$$

and hence

$$
\varphi(y)-\varphi\left(u_{n}\right)+\left\langle y-u_{n_{i}}, \frac{u_{n_{i}}-x_{n_{i}}}{r_{n_{i}}}\right\rangle \geq F\left(y, u_{n_{i}}\right) \quad \forall y \in C .
$$


From $\left\|u_{n}-x_{n}\right\| \rightarrow 0,\left\|x_{n}-W_{n} v_{n}\right\| \rightarrow 0$, and $\left\|W_{n} v_{n}-v_{n}\right\| \rightarrow 0$, we get $u_{n_{i}} \rightarrow v$. Since $\left(u_{n_{i}}-x_{n_{i}}\right) / r_{n_{i}} \rightarrow 0$, it follows by (A4) and the weak lower semicontinuity of $\varphi$ that

$$
F(y, v)+\varphi(v)-\varphi(y) \leq 0 \quad \forall y \in C .
$$

For $t$ with $0<t \leq 1$ and $y \in C$, let $y_{t}=t y+(1-t) v$. Since $y \in C$ and $v \in C$, we have $y_{t} \in C$ and hence $F\left(y_{t}, v\right)+\varphi(v)-\varphi\left(y_{t}\right) \leq 0$. So, from (A1), (A4) and the convexity of $\varphi$, we have

$$
\begin{aligned}
0 & =F\left(y_{t}, y_{t}\right)+\varphi\left(y_{t}\right)-\varphi\left(y_{t}\right) \\
& \leq t F\left(y_{t}, y\right)+(1-t) F\left(y_{t}, v\right)+t \varphi(y)+(1-t) \varphi(v)-\varphi\left(y_{t}\right) \\
& \leq t\left(F\left(y_{t}, y\right)+\varphi(y)-\varphi\left(y_{t}\right)\right) .
\end{aligned}
$$

Dividing by $t$, we get $F\left(y_{t}, y\right)+\varphi(y)-\varphi\left(y_{t}\right) \geq 0$. From (A3) and the weak lower semicontinuity of $\varphi$, we have $F(v, y)+\varphi(y)-\varphi(v) \geq 0$ for all $y \in C$ and hence $v \in M E P(F, \varphi)$.

Next, we show that $v \in F(W)=\bigcap_{n=1}^{\infty} F\left(W_{n}\right)$, where $F\left(W_{n}\right)=\bigcap_{i=1}^{n} F\left(T_{i}\right)$ for every $n \geq 1$, and $F\left(W_{n+1}\right) \subset F\left(W_{n}\right)$. Assume that $v \notin F(W)$, then there exists a positive integer $m$ such that $v \notin F\left(T_{m}\right)$ and so $v \notin \bigcap_{i=1}^{m} F\left(T_{i}\right)$. Hence for any $n \geq m$, $v \notin \bigcap_{i=1}^{n} F\left(T_{i}\right)=F\left(W_{n}\right)$, i.e., $v \neq W_{n} v$. It follows from Opial's condition that

$$
\begin{aligned}
\liminf _{i \rightarrow \infty}\left\|v_{n_{i}}-v\right\| & <\liminf _{i \rightarrow \infty}\left\|v_{n_{i}}-W_{n} v\right\| \\
& \leq \liminf _{i \rightarrow \infty}\left(\left\|v_{n_{i}}-W_{n} v_{n_{i}}\right\|+\left\|W_{n} v_{n_{i}}-W_{n} v\right\|\right) \\
& \leq \liminf _{i \rightarrow \infty}\left\|v_{n_{i}}-v\right\|,
\end{aligned}
$$

which is a contradiction. Thus, we obtain $v \in F(W)$.

Next, we show that $v \in I\left(A_{1}, M_{1}\right)$ and $v \in I\left(A_{2}, M_{2}\right)$. The fact that $A_{1}$ is a $\sigma_{1^{-}}$ inverse-strongly monotone mapping implies that $A_{1}$ is a $\frac{1}{\sigma_{1}}$-Lipschitz continuous monotone mapping and the domain of $A_{1}$ equals $H$. It follows from Lemma 2.10 that $M_{1}+A_{1}$ is maximal monotone. Let $(y, g) \in G\left(M_{1}+A_{1}\right)$, that is, $g-A_{1} y \in M_{1}(y)$. Since $v_{n_{i}}=J_{M_{1}, \tau}\left(y_{n_{i}}-\tau A_{1} y_{n_{i}}\right)$, we have $y_{n_{i}}-\tau A_{1} y_{n_{i}} \in\left(I+\tau M_{1}\right)\left(v_{n_{i}}\right)$, that is,

$$
\frac{1}{\tau}\left(y_{n_{i}}-v_{n_{i}}-\tau A_{1} y_{n_{i}}\right) \in M_{1}\left(v_{n_{i}}\right) .
$$

Since $M_{1}+A_{1}$ is maximal monotone, we have

$$
\left\langle y-v_{n_{i}}, g-A_{1} y-\frac{1}{\lambda}\left(y_{n_{i}}-v_{n_{i}}-\tau A_{1} y_{n_{i}}\right)\right\rangle \geq 0,
$$

and so

$$
\begin{aligned}
\left\langle y-v_{n_{i}}, g\right\rangle & \geq\left\langle y-v_{n_{i}}, A_{1} y+\frac{1}{\tau}\left(y_{n_{i}}-v_{n_{i}}-\tau A_{1} y_{n_{i}}\right)\right\rangle \\
& =\left\langle y-v_{n_{i}}, A_{1} y-A_{1} v_{n_{i}}+A_{1} v_{n_{i}}-A_{1} y_{n_{i}}+\frac{1}{\tau}\left(y_{n_{i}}-v_{n_{i}}\right)\right\rangle \\
& \geq 0+\left\langle y-v_{n_{i}}, A_{1} v_{n_{i}}-A_{1} y_{n_{i}}\right\rangle+\left\langle y-v_{n_{i}}, \frac{1}{\tau}\left(y_{n_{i}}-v_{n_{i}}\right)\right\rangle
\end{aligned}
$$

It follows from $(39)$ and $v_{n_{i}} \rightarrow v$ that

$$
\lim _{i \rightarrow \infty}\left\langle y-v_{n_{i}}, g\right\rangle=\langle y-v, g\rangle \geq 0
$$

It follows from the maximal monotonicity of $M_{1}+A_{1}$ that $\theta \in\left(M_{1}+A_{1}\right)(v)$, that is, $v \in I\left(A_{1}, M_{1}\right)$. By the same way, from (40) and $y_{n_{i}} \rightarrow v$, we obtain $v \in I\left(A_{2}, M_{2}\right)$. Hence 
$v \in \Omega$ is proved.

Since $z=P_{\Omega}(I-B+\gamma f)(z)$, it follows that

$$
\begin{aligned}
\limsup _{n \rightarrow \infty}\left\langle(B-\gamma f) z, z-x_{n}\right\rangle & =\limsup _{n \rightarrow \infty}\left\langle(B-\gamma f) z, z-v_{n}\right\rangle \\
& =\lim _{i \rightarrow \infty}\left\langle(B-\gamma f) z, z-v_{n_{i}}\right\rangle=\langle(B-\gamma f) z, z-v\rangle \leq 0 .
\end{aligned}
$$

By (41), (42) and the last inequality, we conclude that

$$
\limsup _{n \rightarrow \infty}\left\langle\gamma f z-B z, W_{n} v_{n}-z\right\rangle \leq 0 .
$$

Finally, we show that $\left\{x_{n}\right\}$ converges strongly to $z$. Indeed, from (16) we have

$$
\begin{aligned}
& \left\|x_{n+1}-z\right\|^{2} \\
& =\left\|\alpha_{n} \gamma f\left(x_{n}\right)+\beta_{n} x_{n}+\left(\left(1-\beta_{n}\right) I-\alpha_{n} B\right) W_{n} v_{n}-z\right\|^{2} \\
& =\left\|\alpha_{n}\left(\gamma f\left(x_{n}\right)-B z\right)+\beta_{n}\left(x_{n}-z\right)+\left(\left(1-\beta_{n}\right) I-\alpha_{n} B\right)\left(W_{n} v_{n}-z\right)\right\|^{2} \\
& =\alpha_{n}^{2}\left\|\gamma f\left(x_{n}\right)-B z\right\|^{2}+\left\|\beta_{n}\left(x_{n}-z\right)+\left(\left(1-\beta_{n}\right) I-\alpha_{n} B\right)\left(W_{n} v_{n}-z\right)\right\|^{2} \\
& +2\left\langle\beta_{n}\left(x_{n}-z\right)+\left(\left(1-\beta_{n}\right) I-\alpha_{n} B\right)\left(W_{n} v_{n}-z\right), \alpha_{n}\left(\gamma f\left(x_{n}\right)-B z\right)\right\rangle \\
& \leq \alpha_{n}^{2}\left\|\gamma f\left(x_{n}\right)-B z\right\|^{2}+\left(\beta_{n}\left\|x_{n}-z\right\|+\left(1-\beta_{n}-\alpha_{n} \bar{\gamma}\right)\left\|v_{n}-z\right\|\right)^{2} \\
& +2 \alpha_{n} \beta_{n}\left\langle x_{n}-z, \gamma f\left(x_{n}\right)-B z\right\rangle \\
& +2 \alpha_{n}\left(1-\beta_{n}-\alpha_{n} \bar{\gamma}\right)\left\langle W_{n} v_{n}-z, \gamma f\left(x_{n}\right)-B z\right\rangle \\
& \leq \alpha_{n}^{2}\left\|\gamma f\left(x_{n}\right)-B z\right\|^{2}+\left(\beta_{n}\left\|x_{n}-z\right\|+\left(1-\beta_{n}-\alpha_{n} \bar{\gamma}\right)\left\|x_{n}-z\right\|\right)^{2} \\
& +2 \alpha_{n} \beta_{n}\left\langle x_{n}-z, \gamma f\left(x_{n}\right)-\gamma f(z)\right\rangle+2 \alpha_{n} \beta_{n}\left\langle x_{n}-z, \gamma f(z)-B z\right\rangle \\
& +2 \alpha_{n}\left(1-\beta_{n}-\alpha_{n} \bar{\gamma}\right)\left\langle W_{n} v_{n}-z, \gamma f\left(x_{n}\right)-\gamma f(z)\right\rangle \\
& +2 \alpha_{n}\left(1-\beta_{n}-\alpha_{n} \bar{\gamma}\right)\left\langle W_{n} v_{n}-z, \gamma f(z)-B z\right\rangle \\
& \leq \alpha_{n}^{2}\left\|\gamma f\left(x_{n}\right)-B z\right\|^{2}+\left(1-\alpha_{n} \bar{\gamma}\right)^{2}\left\|x_{n}-z\right\|^{2} \\
& +2 \alpha_{n} \beta_{n} \gamma\left\|x_{n}-z\right\|\left\|f\left(x_{n}\right)-f(z)\right\|+2 \alpha_{n} \beta_{n}\left\langle x_{n}-z, \gamma f(z)-B z\right\rangle \\
& +2 \alpha_{n}\left(1-\beta_{n}-\alpha_{n} \bar{\gamma}\right) \gamma\left\|W_{n} v_{n}-z\right\|\left\|f\left(x_{n}\right)-f(z)\right\| \\
& +2 \alpha_{n}\left(1-\beta_{n}-\alpha_{n} \bar{\gamma}\right)\left\langle W_{n} v_{n}-z, \gamma f(z)-B z\right\rangle \\
& \leq \alpha_{n}^{2}\left\|\gamma f\left(x_{n}\right)-B z\right\|^{2}+\left(1-\alpha_{n} \bar{\gamma}\right)^{2}\left\|x_{n}-z\right\|^{2} \\
& +2 \alpha_{n} \beta_{n} \gamma \alpha\left\|x_{n}-z\right\|^{2}+2 \alpha_{n} \beta_{n}\left\langle x_{n}-z, \gamma f(z)-B z\right\rangle \\
& +2 \alpha_{n}\left(1-\beta_{n}-\alpha_{n} \bar{\gamma}\right) \gamma \alpha\left\|x_{n}-z\right\|^{2} \\
& +2 \alpha_{n}\left(1-\beta_{n}-\alpha_{n} \bar{\gamma}\right)\left\langle W_{n} v_{n}-z, \gamma f(z)-B z\right\rangle \\
& =\alpha_{n}^{2}\left\|\gamma f\left(x_{n}\right)-B z\right\|^{2}+\left(1-2 \alpha_{n} \bar{\gamma}+\alpha_{n}^{2} \bar{\gamma}^{2}-2 \alpha_{n}^{2} \bar{\gamma} \gamma \alpha\right)\left\|x_{n}-z\right\|^{2} \\
& +2 \alpha_{n} \beta_{n}\left\langle x_{n}-z, \gamma f(z)-B z\right\rangle+2 \alpha_{n}\left(1-\beta_{n}-\alpha_{n} \bar{\gamma}\right)\left\langle W_{n} v_{n}-z, \gamma f(z)-B z\right\rangle \\
& \leq\left(1-\alpha_{n}\left(2 \bar{\gamma}+\alpha_{n} \bar{\gamma}^{2}\right)\right)\left\|x_{n}-z\right\|^{2}+2 \alpha_{n} \sigma_{n}
\end{aligned}
$$

where $\sigma_{n}=\alpha_{n}\left\|\gamma f\left(x_{n}\right)-B z\right\|^{2}+2 \beta_{n}\left\langle x_{n}-z, \gamma f(z)-B z\right\rangle+2\left(1-\beta_{n}-\alpha_{n} \bar{\gamma}\right) \times$ $\left\langle W_{n} v_{n}-z, \gamma f(z)-B z\right\rangle$. By (48), we get $\limsup _{n \rightarrow \infty} \sigma_{n} \leq 0$. Hence by Lemma 2.5 applied to (49), we conclude that $x_{n} \rightarrow z$. This completes the proof.

Using Theorem 3.1, we obtain the following corollaries. 
Corollary 3.2. Let $C$ be a nonempty closed convex subset of a real Hilbert space H. Let $F$ be a bifunction of $C \times C$ into real numbers $\mathbf{R}$ satisfying (A1)-(A5), $f$ be a contraction of $H$ into itself with coefficient $\alpha \in(0,1)$ and $B$ be a strongly bounded linear operator on $H$ with coefficient $\bar{\gamma}>0$ and $0<\gamma<\frac{\bar{\gamma}}{\alpha}$. Let $M: H \rightarrow 2^{H}$ be a maximal monotone mapping and $A: H \rightarrow H$ a $\sigma$-inverse-strongly monotone mapping. Let $\left\{V_{i}: C \rightarrow C\right\}_{i=1}^{\infty}$ be a countable family of uniformly $k$-strict pseudo-contractions, $\left\{T_{i}: C \rightarrow C\right\}_{i=1}^{\infty}$ be the countable family of nonexpansive mappings defined by $T_{i} x=t x+(1-t) V_{i} x$, for $x \in C$, $i \geq 1, t \in[k, 1)$. Let $W_{n}$ be the $W$-mapping defined by (14) and $W$ be a mapping defined by (15) with $F(W) \neq \emptyset$. Assume that $\Omega:=\bigcap_{n=1}^{N} F\left(T_{i}\right) \cap I(A, M) \cap E P(F) \neq \emptyset$. Let $\left\{x_{n}\right\},\left\{y_{n}\right\},\left\{z_{n}\right\}$ and $\left\{u_{n}\right\}$ be sequences generated by $x_{1} \in H$ and

$$
\left\{\begin{array}{l}
F\left(u_{n}, y\right)+\frac{1}{r_{n}}\left\langle y-u_{n}, u_{n}-x_{n}\right\rangle \geq 0 \quad \forall y \in C \\
y_{n}=J_{M, \tau}\left(u_{n}-\tau A u_{n}\right) \\
v_{n}=J_{M, \tau}\left(y_{n}-\tau A y_{n}\right) \\
x_{n+1}=\alpha_{n} \gamma f\left(x_{n}\right)+\beta_{n} x_{n}+\left(\left(1-\beta_{n}\right) I-\alpha_{n} B\right) W_{n} v_{n}
\end{array}\right.
$$

for every $n \geq 1$, where $\left\{\alpha_{n}\right\},\left\{\beta_{n}\right\} \subset(0,1),\left\{r_{n}\right\} \subset(0, \infty)$ and $\tau \in(0,2 \sigma)$ satisfy conditions (i)-(iii) in Theorem 3.1. Then $\left\{x_{n}\right\}$ converges strongly to $z \in \Omega$ which is the unique solution of the variational inequality

$$
\langle(B-\gamma f) z, z-x\rangle \leq 0, \quad x \in \Omega .
$$

Equivalently, we have $z=P_{\Omega}(I-B+\gamma f)(z)$.

Proof. Taking $\varphi \equiv 0, M_{1}=M_{2}=M, A_{1}=A_{2}=A$ and $\tau=\delta$ in Theorem 3.1, we obtain the desired conclusion easily. This completes the proof.

Corollary 3.3. Let $C$ be a nonempty closed convex subset of a real Hilbert space $H$. Let $F$ be a bifunction of $C \times C$ into real numbers $\mathbf{R}$ satisfying (A1)-(A5) and let $\varphi: C \rightarrow$ $\mathbf{R} \cup\{+\infty\}$ be a proper lower semicontinuous and convex function. Let $f$ be a contraction of $H$ into itself with coefficient $\alpha \in(0,1)$ and let $B$ be a strongly bounded linear operator on $H$ with coefficient $\bar{\gamma}>0$ and $0<\gamma<\frac{\bar{\gamma}}{\alpha}$. Let $A_{1}, A_{2}: H \rightarrow H$ be $\sigma_{1}, \sigma_{2}$-inverse-strongly monotone mappings, respectively. Let $\left\{V_{i}: C \rightarrow C\right\}_{i=1}^{\infty}$ be a countable family of uniformly $k$-strict pseudo-contractions, $\left\{T_{i}: C \rightarrow C\right\}_{i=1}^{\infty}$ be the countable family of nonexpansive mappings defined by $T_{i} x=t x+(1-t) V_{i} x$, for $x \in C, i \geq 1, t \in[k, 1)$. Let $W_{n}$ be the $W$-mapping defined by (14) and $W$ be a mapping defined by (15) with $F(W) \neq \emptyset$. Assume that either (B1) or (B2) holds and $\Omega:=\bigcap_{n=1}^{N} F\left(T_{i}\right) \cap V I\left(C, A_{1}\right) \cap V I\left(C, A_{2}\right) \cap$ $\operatorname{MEP}(F, \varphi) \neq \emptyset$. Let $\left\{x_{n}\right\},\left\{y_{n}\right\},\left\{z_{n}\right\}$ and $\left\{u_{n}\right\}$ be sequences generated by $x_{1} \in H$ and

$$
\left\{\begin{array}{l}
F\left(u_{n}, y\right)+\varphi(y)-\varphi\left(u_{n}\right)+\frac{1}{r_{n}}\left\langle y-u_{n}, u_{n}-x_{n}\right\rangle \geq 0 \quad \forall y \in C, \\
y_{n}=P_{C}\left(u_{n}-\delta A_{2} u_{n}\right), \\
v_{n}=P_{C}\left(y_{n}-\tau A_{1} y_{n}\right), \\
x_{n+1}=\alpha_{n} \gamma f\left(x_{n}\right)+\beta_{n} x_{n}+\left(\left(1-\beta_{n}\right) I-\alpha_{n} B\right) W_{n} v_{n},
\end{array}\right.
$$

for every $n \geq 1$, where $\left\{\alpha_{n}\right\},\left\{\beta_{n}\right\} \subset(0,1),\left\{r_{n}\right\} \subset(0, \infty), \tau \in\left(0,2 \sigma_{1}\right)$ and $\delta \in\left(0,2 \sigma_{2}\right)$ satisfy conditions (i)-(iii) in Theorem 3.1. Then $\left\{x_{n}\right\}$ converges strongly to $z \in \Omega$ which 
is the unique solution of the variational inequality

$$
\langle(B-\gamma f) z, z-x\rangle \leq 0, \quad x \in \Omega .
$$

Equivalently, we have $z=P_{\Omega}(I-B+\gamma f)(z)$.

Proof. In Theorem 3.1 put $M_{1}=M_{2}=\partial \delta_{C}$, then $J_{M_{1}, \tau}=J_{M_{2}, \delta}=P_{C}$. The conclusion can be obtained immediately.

Corollary 3.4. Let $C$ be a nonempty closed convex subset of a real Hilbert space $H$. Let $f$ be a contraction of $H$ into itself with coefficient $\alpha \in(0,1)$ and let $B$ be a strongly bounded linear operator on $H$ with coefficient $\bar{\gamma}>0$ and $0<\gamma<\frac{\bar{\gamma}}{\alpha}$. Let $M_{1}, M_{2}: H \rightarrow 2^{H}$ be maximal monotone mappings and $A_{1}, A_{2}: H \rightarrow H$ be $\sigma_{1}, \sigma_{2}$-inverse-strongly monotone mappings, respectively. Let $\left\{V_{i}: C \rightarrow C\right\}_{i=1}^{\infty}$ be a countable family of uniformly $k$-strict pseudo-contractions, $\left\{T_{i}: C \rightarrow C\right\}_{i=1}^{\infty}$ be the countable family of nonexpansive mappings defined by $T_{i} x=t x+(1-t) V_{i} x$ for $x \in C, i \geq 1, t \in[k, 1)$. Let $W_{n}$ be the $W$ mapping defined by (14) and $W$ be a mapping defined by $(15)$ with $F(W) \neq \emptyset$. Assume that $\Omega:=\bigcap_{n=1}^{N} F\left(T_{i}\right) \cap I\left(A_{1}, M_{1}\right) \cap I\left(A_{2}, M_{2}\right) \neq \emptyset$. Let $\left\{x_{n}\right\},\left\{y_{n}\right\},\left\{z_{n}\right\}$ and $\left\{u_{n}\right\}$ be sequences generated by $x_{1} \in H$ and

$$
\left\{\begin{array}{l}
y_{n}=J_{M_{2}, \delta}\left(x_{n}-\delta A_{2} x_{n}\right), \\
v_{n}=J_{M_{1}, \tau}\left(y_{n}-\tau A_{1} y_{n}\right), \\
x_{n+1}=\alpha_{n} \gamma f\left(x_{n}\right)+\beta_{n} x_{n}+\left(\left(1-\beta_{n}\right) I-\alpha_{n} B\right) W_{n} v_{n},
\end{array}\right.
$$

for every $n \geq 1$, where $\left\{\alpha_{n}\right\},\left\{\beta_{n}\right\} \subset(0,1), \tau \in\left(0,2 \sigma_{1}\right)$ and $\delta \in\left(0,2 \sigma_{2}\right)$ satisfy conditions (i),(iii) in Theorem 3.1. Then $\left\{x_{n}\right\}$ converges strongly to $z \in \Omega$ which is the unique solution of the variational inequality

$$
\langle(B-\gamma f) z, z-x\rangle \leq 0, \quad x \in \Omega .
$$

Equivalently, we have $z=P_{\Omega}(I-B+\gamma f)(z)$.

Proof. Putting in Theorem $3.1 \varphi \equiv 0$ and $F(x, y)=0$ for all $x, y \in C$, we deduce that $u_{n}=P_{C} x_{n}=x_{n}$. Thus the desired conclusion follows easily. This completes the proof.

Acknowledgments. The first author would like to thank the Faculty of Science, King Mongkut's University of Technology Thonburi (KMUTT) for their financial support during the preparation of this paper and the second author would like to thank the Thailand Research Fund and the Commission on Higher Education for Grant No. MRG5380044. Moreover, we would like to thank the organizing committee of the Function Spaces IX for publication of our paper.

\section{References}

[BO] E. Blum, W. Oettli, From optimization and variational inequalities to equilibrium problems, Math. Student 63 (1994), 123-145.

[Bré] H. Brézis, Opérateurs maximaux monotones et semi-groupes de contractions dans les espaces de Hilbert, North-Holland Math. Studies 5. Notas de Matemática (50), North-Holland, Amsterdam, 1973. 
[Bro] F. E. Browder, Nonlinear monotone operators and convex sets in Banach spaces, Bull. Amer. Math. Soc. 71 (1965), 780-785.

[CY] L. C. Ceng, J. C. Yao, A hybrid iterative scheme for mixed equilibrium problems and fixed point problems, J. Comput. Appl. Math. 214 (2008), 186-201.

[C] S. S. Chang, Variational Inequalities and Related Problems, Chongqing Publ. House, 2007 (in Chinese).

[FA] S. D. Flåm, A. S. Antipin, Equilibrium programming using proximal-like algorithms, Math. Programming 78 (1997), 29-41.

[HS] P. Hartman, G. Stampacchia, On some nonlinear elliptic differential equations, Acta Math. 115 (1966), 271-310.

[K1] P. Kumam, A hybrid approximation method for equilibrium and fixed point problems for a monotone mapping and a nonexpansive mapping, Nonlinear Anal. Hybrid Syst. 2 (2008), 1245-1255.

[K2] P. Kumam, A new hybrid iterative method for solution of equilibrium problems and fixed point problems for an inverse strongly monotone operator and a nonexpansive mapping, J. Appl. Math. Comput. 29 (2009), 263-280.

[K3] P. Kumam, Strong convergence theorems by an extragradient method for solving variational inequalities and equilibrium problems in a Hilbert space, Turkish J. Math. 33 (2009), 85-98.

[KK] P. Kumam, P. Katchang, A viscosity of extragradient approximation method for finding equilibrium problems, variational inequalities and fixed point problems for nonexpansive mappings, Nonlinear Anal. Hybrid Syst. 3 (2009), 475-486.

[LS] J.-L. Lions, G. Stampacchia, Variational inequalities, Comm. Pure Appl. Math. 20 (1967), 493-517.

[MX] G. Marino, H.-K. Xu, A general iterative method for nonexpansive mappings in Hilbert spaces, J. Math. Anal. Appl. 318 (2006), 43-52.

[MT] A. Moudafi, M. Théra, Proximal and dynamical approaches to equilibrium problems, in: Ill-posed Variational Problems and Regularization Techniques, Lecture Notes in Econom. and Math. Systems 477, Springer, Berlin, 1999, 187-201.

[O] Z. Opial, Weak convergence of the sequence of successive approximation for nonexpansive mapping, Bull. Amer. Math. Soc. 73 (1967), 591-597.

[OI] M. O. Osilike, D. I. Igbokwe, Weak and strong convergence theorems for fixed points of pseudocontractions and solutions of monotone type operator equations, Comput. Math. Appl. 40 (2000), 559-567.

[PWSY] J.-W. Peng, Y. Wang, D. S. Shyu, J.-C. Yao, Common solutions of an iterative scheme for variational inclusions, equilibrium problems and fixed point problems, J. Inequal. Appl. 2008, Article ID 720371, 15 pp.

[PY] J.-W. Peng, J.-C. Yao, Strong convergence theorems of iterative scheme based on the extragradient method for mixed equilibrium problems and fixed point problems, Math. Comput. Modelling 49 (2009), 1816-1828.

[PS] S. Plubtieng, W. Sriprad, A viscosity approximation method for finding common solutions of variational inclusions, equilibrium problems and fixed point problems in Hilbert spaces, Fixed Point Theory Appl. 2009, Article ID 567147, 20 pp.

[ST] K. Shimoji, W. Takahashi, Strong convergence to common fixed points of infinite nonexpansive mappings and applications, Taiwanese J. Math. 5 (2001), 387-404. 
[S] T. Suzuki, Strong convergence of Krasnoselskii and Mann's type sequences for oneparameter nonexpansive semigroups without Bochner integrals, J. Math. Anal. Appl. 305 (2005), 227-239.

[X] H. K. Xu, Viscosity approximation methods for nonexpansive mappings, J. Math. Anal. Appl. 298 (2004), 279-291.

[ZLC] S.-S. Zhang, J. H. W. Lee, C. K. Chan, Algorithms of common solutions to quasi variational inclusion and fixed point problems, Appl. Math. Mech. (English Ed.) 29 (2008), 571-581.

[Z] H. Zhou, Convergence theorems of fixed points for $\kappa$-strict pseudo-contractions in Hilbert spaces, Nonlinear Anal. 69 (2008), 456-462. 